\title{
Capítulo V - Educação em asma
}

A educação é fundamental para o sucesso do controle da asma, tendo um impacto positivo na mudança ativa de comportamento frente à doença.

Existe ainda muita diversidade nos programas educati$\operatorname{vos}^{(1,2)}$.

Os principais níveis de educação estão indicados no quadro abaixo(3-19).

\section{CONTEÚdo dos programas}

O Quadro 2 resume as metas a serem cumpridas pelo profissional da saúde ao orientar sobre Educação em Asma(1).

\section{Elaboração de um plano de aÇão}

O Plano de ação deve ser por escrito e constitui o ponto central do tratamento(1). Deve ser individualizado e elaborado pelo médico em parceria com o paciente, conforme o Quadro 3.

\section{Ferramentas de aplicaÇão}

Pacientes asmáticos requerem acompanhamento regular após o início da terapia a intervalos programados, dependendo da gravidade da asma(4,14).

Os programas podem ser aplicados a indivíduos ou a grupos por equipes multidisciplinares.

\section{QUADRO 1 \\ Principais níveis de educação em asma}

- Educar os profissionais ligados à saúde

- Diagnóstico correto e precoce

- Adesão e terapêutica adequadas

- Atualização e aplicação dos consensos

- Educar os asmáticos

- Informativo: simples informação oral ou escrita

b Aumenta a percepção dos sintomas, mas não melhora os indicadores de asma

- Estruturados: educação do paciente, monitorização da doença, revisão médica e plano de ação por escrito

b Reduz o número de hospitalizações, visitas ao pronto-socorro, visitas não agendadas ao ambulatório e o absenteísmo. Sem efeito para episódios de asma noturna, $\mathrm{VEF}_{1}$, PFE e cursos de corticosteróide oral

\section{QUADRO 2}

Metas do programa de educação em asma

Explicar a cronicidade da asma e o reconhecimento dos sintomas

- Identificar fatores agravantes e orientar como evitá-los

- U sar medicamentos apropriados e com técnica adequada

- Ensinar a execução de um plano de ação
QUADRO 3

Itens de um plano de ação adequado

- Monitorização dos sintomas da asma e/ou do PFE

- Especificação do tratamento de manutenção

- Reconhecimento dos sinais e sintomas precoces de exacerbação

- Proposta de alteração do esquema terapêutico

- Tratamento domiciliar das crises leves

- Indicações claras de quando procurar um serviço de emergência 
Os programas estruturados de longo prazo, no mínimo seis meses, mais freqüentemente 12 meses ou mais, demonstram melhores resultados.

Os programas de intervenção geralmente apresentam melhores resultados, se especificamente criados para a classe a que se destinam $(4,13,14)$.

\section{ReferÊnCIAS}

1. Fernandes ALG, Cabral ALB, Faresin SM. I Consenso Brasileiro de Educação em Asma. J Pneumol 1996;22(Supl):1-24.

2. Sudre $P$, J acquemet $S$, Uldry $C$, Perneger TV. Objectives, methods and content of patient education programmes for adults with asthma: systematic review of studies published between 1979 and 1998. Thorax 1999;54:681-7

3. Abramson MJ, Haydn W and the Victorian Asthma Mortality Study Group, Bailey MJ, Couper FJ, Driver J S, Drummer OI, Forbes AB, $\mathrm{MCN}$ eil J I. Are asthma medications and management related to deaths from asthma? Am J Respir Crit Care Med 2001;163:12-8.

4. Cabral ALB, Martins MA, Carvalho WAF, Chinen M, Barbirotto RM, Boueri FMV. Are international asthma guidelines effective for low-income Brazilian children with asthma? Eur Respir J 1998;12:35-40.

5. Chafin CC, Toley E, Demirkan K, Self TH. Effect of a brief educational intervention on medical students use of asthma devices. J Asthma 2000; 37:585-8.

6. Cote J, Boulet LP, Cartier A, Robichaud P, Boutin H, Malo J L, et al Influence of asthma education on asthma severity, quality of life and environmental control. Can Respir J 2000;7:395-400.

7. Cote J, Boulet LP, Bowie DM, Robichaud P, Parent J G, Battisti L. Evaluation of two different educational interventions for adult patients consulting with an acute asthma exacerbation. Am J Respir Crit Care Med 2001;163:1415-9.

8. Cowie RL, Underwood MF, Mack S. The impact of asthma management guideline dissemination on the control of asthma in the community. Can Respir J 2001;8(Suppl A):41A-5A.
9. Gibson PG, Walters EH, Coughlanj, Wilson AJ, Hensley MJ, Abramson $M$, et al. Limited (information only) patient education programs for adults with asthma. Cochrane Database Syst Rev CD001005 2000;2.

10. Green RJ , Adam B, Greenblatt MM, Plit M, J ones S. Asthma management and perceptions in rural South Africa. Ann Allergy Asthma Immunol 2001;86:343-7.

11. Homer C, Rubin DH, Susskind O, Alpert HR, O wusu MS, Schneider $L$, et al. An evaluation of an innovative multimedia educational software program for asthma management: report of a randomized, controlled trial. Pediatrics 2000;106(pt2):210-5.

12. Liu C, Feekery C. Can asthma education improve clinical outcomes? An evaluation of a pediatric asthma education program. J Asthma 2001; 38:269-78.

13. Oliveira MA, Fernandes ALG, Bruno VF, Ballini LS, J ardim J RB. Evaluation of an educational program for asthma control in adults. J Asthma 1997;35:395-403.

14. Oliveira MA, Fernandes ALG, Faresin SM, Bruno VF, Bittencourt AR. Evaluation of an educational program for socially deprived asthma patients. Eur Resp J 1999;14:908-14.

15. Peterson MW, Strommer-Pace L, Dayton C. Asthma patient education: current utilization in pulmonary training programs. J Asthma 2001; 38:261-7.

16. Shah S, Gibson PG, Peat JK, Mazurski EJ, Wang H, Sindhusake D, et al. Effect of peer led programme for asthma education in adolescents: cluster randomized controlled trial. BMJ 2001;322:583-5.

17. Shegog R, Abramson SL, Bartholomew LK, Parcel GS, Socckrider MM, Mâsse L. Impact of a computer-assisted education program on factors related to asthma self-management behavior. J Am Med Inform Assoc 2001;8:49-61.

18. Vieira JE, Cukier A, Stelmach R, Kasahara DI, Gannam S, Warth MD. Comparison of knowledge on asthma: doctors completing internal medicine residency and doctors completing medical school. Sao Paulo Med J 2001;119:101-4.

19. Yawn BP, Markson L, Algatt-Bergstrom PJ, Yawn RA, Wollan P, Grecco $M$, et al. An in school CD-ROM asthma educational program. J Sch Health 2000;70:153-9. 